of CT infection is distinct from women with persisting chlamydia. These studies may inform whether IFN- $\gamma$, produced by CD4+ T-cells, or tryptophan-dependent or -independent metabolic pathways are associated with natural clearance, which may advance chlamydia vaccine development.

\section{Serial Biomarker Monitoring Predicts Long Term Outcomes in Acute Graft Versus Host Disease}

Hrishikesh Krishna Srinagesh ${ }^{1}$, Hrishikesh Krishna Srinagesh, Urvi Kapoor, Mina Aziz, Kaitlyn Ben-David, Hannah Major-Monfried, George Morales, Rachel Young, Umut Ozbek, John E Levine and James LM Ferrara

${ }^{1}$ Mount Sinai School of Medicine

OBJECTIVES/SPECIFIC AIMS: The first aim of the study is to evaluate the accuracy of serum biomarkers of acute GVHD measured after four weeks of corticosteroid therapy to predict 6 month NRM. The second aim of this study is to compare the accuracy of the biomarker algorithm to that of clinical response to corticosteroids after four weeks. The third aim of the study is to develop a novel regression model that uses weekly biomarker measurements over the first month of corticosteroid therapy to predict 6 month NRM. METHODS/STUDY POPULATION:. Patients who received HCT at one of 22 IRB-approved centers and provided blood samples to the Mount Sinai Acute GVHD International Consortium (MAGIC) biorepository and developed GVHD between January 2008 to May 2018 are included in this study. Patients were divided by time into a training set (Jan 2008-Dec 2015, n=233) for model development and a validation set (Jan 2015-May 2018, $n=357$ ) to evaluate the predictive performance of the model. The later time of the validation set was chosen deliberately to model contemporaneous GVHD treatment practices. The size of each group was designed so that there would be roughly equal numbers of deaths in both groups. RESULTS/ANTICIPATED RESULTS:. Serum concentrations of GVHD biomarkers after one month of corticosteroid therapy were measured in the validation set, and the predicted probability of NRM $(\hat{\mathrm{p}})$ was computed according to the previously published algorithm: $\log [-\log (1-\hat{\mathrm{p}})]=-11.263+1.844(\operatorname{logST} 2)+0.577(\operatorname{logREG} 3 \alpha)$. The performance of the biomarker algorithm was evaluated by creating receiver operating characteristic (ROC) curves and calculating the area under the curve (AUC) in the validation set. The AUC of the biomarker algorithm was a significantly better predictor of 6 month NRM than clinical response to treatment after four weeks of corticosteroids $(0.84$ vs. $0.64, \mathrm{p}<0.001)$, which is a clinically relevant improvement in accuracy. To evaluate serial biomarker monitoring, serum biomarker concentrations will be measured weekly at five time points from treatment initiation to one month after corticosteroid therapy. We will use these values in the training set to develop a regression model for 6 month NRM that accounts for repeated biomarker measurements. The performance of this model will be tested in the validation set and the accuracy of the serial biomarker measurements will be compared to the accuracy of measuring biomarkers at the single time point after four weeks of corticosteroid therapy. An AUC improvement of 0.05 would be considered clinically significant. DISCUSSION/SIGNIFICANCE OF IMPACT: Clinical response to treatment after four weeks has been the standard endpoint in GVHD interventional trials for decades. If biomarkers measured at the same time more accurately predict long term mortality, this study would provide the basis for a novel endpoint in GVHD trials and enable more accurate determination of effect size of experimental interventions. An accurate biomarker algorithm will prove useful in guiding immunosuppressive treatment decisions for patients with GVHD. Patients identified by the algorithm as low-risk may benefit from reduced-dose corticosteroid therapy, potentially reducing lethal opportunistic infections. Patients identified as highrisk will be candidates for more intensive immunosuppression or investigational therapies. This precision medicine approach tailors therapy to the individual patient's biology.

3158

\section{Sunitinib-Induced Cardiotoxicity in an Engineered Cardiac Microtissue Model}

Carissa Livingston ${ }^{1}$, Abhinay Ramachandran, Elise Corbin, Alexia Vite, Alexander Bennett and Kenneth Margulies

${ }^{1}$ University of Pennsylvania School of Medicine

OBJECTIVES/SPECIFIC AIMS: The aims of this study are threefold. Firstly, we are examining the effects of increased in vitro afterload (a proxy for hypertension) on human induced pluripotent stem cell cardiomyocyte (hiPSC-CM) response to sunitinib in a durable and dynamic cardiac microtissue culture system. Secondly, we are exploring effects of repeat exposure and recovery of both sunitinib and afterload throughout the lifetime of the hiPSC-CM microtissue. Finally, we are assessing methods to prevent and treat sunitinib induced cardiotoxicity. Primary outcomes for this study are commonly utilized metrics of cardiotoxicity: degree of caspase activation, electrophysiology benchmarks for minimum voltage threshold and maximum capture rate, and microtissue force generation. METHODS/STUDY POPULATION: HiPSC-CMs are cultured and matured as 3D cardiac microtissues (CMTs) on a microtissue array. After maturation, cells are exposed to sunitinib doses of $0 \mu \mathrm{M}, 0.5 \mu \mathrm{M}$, $1 \mu \mathrm{M}$ or $5 \mu \mathrm{M}$ for 12 hours. Concurrently with sunitinib dosing, increases in microtissue array stiffness are created with application of an external magnetic field. Afterload spring constants are fixed at pre-determined physiologic values ranging from $0.5 \mu \mathrm{N} / \mu \mathrm{m}$, to $5 \mu \mathrm{N} / \mu \mathrm{m}$. For Aim 1: Half of the CMTs are harvested at 8 hours after sunitinib dosing to conduct the caspase $3 / 7$ assay, and the remainder are examined for 3 days following drug exposure to track temporal changes in electrophysiology and force generation. For Aim 2: After CMT maturation, 12-hour exposures to sunitinib are repeated three times at a fixed dose, with doses separated by one week. Concurrently with sunitinib dosing, increases or decreases in microtissue stiffness are created by changing the strength of an applied external magnetic field to create "ramp up" or "ramp down" stiffness conditions. Caspase assay and contractility metrics are measured at each timepoint. For Aim 3: Experimental conditions are conducted as described in Aim 1. Prior to the introduction of sunitinib, either carvedilol or an AMP-kinase activator is added to the CMT culture media at physiologic concentrations. Primary outcomes are examined as in Aim 1. RESULTS/ANTICIPATED RESULTS: Aim 1: We hypothesize that increases in microtissue afterload, synchronized with sunitinib exposure will augment sunitinib toxicity in cardiomyocytes resulting in elevations of caspase $3 / 7$ activity and minimum voltage capture as well as decreases in maximum capture rate and maximum force generation. Aim 2: We hypothesize that repeat 
exposures to both sunitinib and to increases in afterload will augment sunitinib toxicity in CMTs via the primary outcomes mentioned in Aim 1. Additionally, we hypothesize that decreases in afterload will decrease effective sunitinib toxicity in CMTs via the primary outcomes mentioned in Aim 1. Aim 3: We hypothesize that exposure to an AMP-kinase activator but not carvedilol will decrease the effects of sunitinib toxicity in CMTs via the primary outcomes mentioned in Aim 1. DISCUSSION/SIGNIFICANCE OF IMPACT: The use of small molecule, targeted chemotherapeutic agents is increasingly common. Many of these agents cause cardiotoxic side effects, the mechanisms of which are incompletely understood. Our lab has developed a novel 3D tissue engineering platform capable of supporting durable in vitro cardiac microtissues that experience dynamic alterations in their biomechanical load. By using this platform to examine the cardiotoxic effects of sunitinib, insight into treatment and prevention of this common problem will be developed.

\section{7}

\section{The effect of common genetic variants in the oxytocin} receptor gene on oxytocin response.

Manasi Malik ${ }^{1}$, Naiqi Shi, Geraldine Serwald, Grace Y. Lee, Antonina I. Frolova, Céline Galés and Sarah K. England

${ }^{1}$ Washington University in St. Louis

OBJECTIVES/SPECIFIC AIMS: Previous studies suggest that genetic variants in the oxytocin receptor (OXTR) may alter oxytocin dose requirement for labor induction and may increase risk for preterm labor and neurodevelopmental disorders. However, the mechanisms of actions of these variants remain unknown. The goal of this study was to functionally characterize common missense and noncoding variants in OXTR. First, we aimed to determine the effects of missense variants on two major aspects of receptor function: calcium signaling and $\beta$-arrestin recruitment. Second, we used allelic expression imbalance assays in an effort to identify regulatory single nucleotide polymorphisms (SNPs) in noncoding regions of OXTR that alter OXTR mRNA expression. METHODS/STUDY POPULATION: We used the Exome Aggregation Consortium database to identify the 12 most prevalent missense single nucleotide variants in OXTR. To determine the functional effects of these variants, we transfected human embryonic kidney cells (a common model system used to study receptor function) with wild type OXTR, variant OXTR, or empty vector control. We used the calcium-sensitive dye Fluo4 to quantify intracellular calcium flux in response to oxytocin treatment, and used bioluminescence resonance energy transfer assays to measure recruitment of the signaling partner $\beta$-arrestin to the receptor. To investigate potential effects of noncoding SNPs on OXTR mRNA expression, we quantified allele-specific expression of OXTR in human uterine tissue obtained from participants at the time of Cesarean section. We used next-generation sequencing (Illumina MiSeq) to count alleles of a reporter SNP in OXTR exon 3. RESULTS/ANTICIPATED RESULTS: Of the 12 most prevalent missense single nucleotide variants, four were predicted to be deleterious by PolyPhen variant annotation software. We anticipate that these variants will alter receptor signaling through calcium or $\beta$-arrestin pathways. We further observed that a reporter SNP in OXTR exon 3 exhibits significant allelic expression imbalance in a subset of our myometrial tissue samples, indicating that OXTR expression may be regulated by a functional SNP. Our current work focuses on discovering the functional SNPs in OXTR responsible for the pattern of allelic expression imbalance seen in mRNA. In the future, we will seek to explore the effects of these variants on uterine function by using genome editing of uterine smooth muscle cells. DISCUSSION/SIGNIFICANCE OF IMPACT: Our results suggest that both missense and noncoding variants may affect OXTR expression and function. Future studies may suggest that OXTR sequencing, genotyping, or expression analysis would be useful to identify individuals likely to respond or fail to respond to safe doses of oxytocin for labor induction. Personalizing approaches for labor induction in this way would increase the safety of oxytocin and potentially reduce maternal morbidity and mortality.

The Effects of Aging on the Rectal Mucosal CD4 $+\mathrm{T}$ cell Compartment and its Implications for HIV Transmission Cassie Grimsley Ackerley ${ }^{1}$, Praveen Kumar Amancha, Phillip M. Murray, Jasper Barnes and Colleen F. Kelley ${ }^{1}$ Emory University

OBJECTIVES/SPECIFIC AIMS: In the first aim, we will evaluate the proportion of highly HIV-susceptible memory CD4+ T cells present in the rectal mucosa, based on the proliferation status and expression of the HIV susceptibility markers, CCR 5 and $\alpha 4 \beta 7$, between HIVnegative adolescent MSM and adult MSM engaging in RAI. The second aim will assess differences between the two study groups in the ratio of Th17 cells $(\mathrm{CD} 4+\mathrm{IL} 17+)$ to Treg cells $(\mathrm{CD} 4+$ FoxP3+ $\mathrm{CD} 25+$ ) in the rectal mucosa as a determinant of mucosal inflammation. Finally, in the third aim, we will utilize ex vivo rectal biopsy explant challenge experiments to examine whether HIV target cell availability and the Th17/Treg ratio influence rectal mucosal HIV susceptibility. METHODS/STUDY POPULATION: Rectal biopsy specimens are being collected from healthy, HIV-negative men that comprise the two study groups: 40 adolescent MSM 18-21 years of age who have engaged in RAI at least once previously in their lifetime and 40 adult $\mathrm{MSM} \geq 35$ years of age who have engaged in RAI for the previous 5 consecutive years with a minimum of 12 episodes annually. To identify CD4+ subsets of interest for aims 1 and 2, rectal mucosal mononuclear cells are isolated and phenotyped with CD45, CD3, CD4, CD45RA, CCR7, CD69, CCR5, $\alpha 4 \beta 7$, Ki67, FOXP3, and CD25 antibodies. To identify the Th17 cell subtype, the cells are stimulated with PMA/Ionamycin and stained with an antibody specific to IL-17A. Using cross-sectional analyses, we will compare the frequencies of mucosal CD4 $+\mathrm{T}$ cells that express certain phenotypic characteristics and evaluate differences in the Th17/Treg ratio between adolescent and adult MSM. For aim 3, rectal biopsy specimens are inoculated with HIV virus and the culture supernatant is assayed for p24 concentration on days 3, 7, 1014 , and 18 . Longitudinal analyses will be performed to detect differences in p24 concentration at each time point and assess associations with mucosal target cell availability and with the Th17/Treg ratio. RESULTS/ANTICIPATED RESULTS: We hypothesize that younger age will be associated with enhanced memory CD4 $+\mathrm{T}$ cell proliferation and increased expression of HIV susceptibility markers (CCR5 and/or $\alpha 4 \beta 7$ ). In addition, we expect that the rectal mucosa of adolescent MSM will demonstrate a higher Th17/Treg ratio as compared to adult MSM, which could facilitate HIV transmission. It is also anticipated that rectal mucosal immune phenotypes characterized by increased HIV target cell availability and high Th17/Treg ratios 